\title{
Refrigeração e cera na qualidade pós-colheita da tangerina 'Fremont' IAC-543
}

\author{
Mariane Aparecida Barbará ${ }^{1}$, Julia Claudiane da Veiga ${ }^{1}$, Bárbara Marçon Pereira da Silva ${ }^{1}$, \\ Sílvia Regina de Toledo Valentini ${ }^{2}$ Patrícia $\mathrm{Cia}^{2}$ \& Ilana Urbano Bron ${ }^{2}$
}

\begin{abstract}
RESUMO
As técnicas de pós-colheita adequadas são fundamentais para o sucesso do armazenamento, e a associação dessas técnicas pode trazer efeito aditivo na manutenção da qualidade dos frutos, como no caso da tangerina 'Fremont' IAC-543, com grande potencial para comercialização. O objetivo do trabalho foi avaliar o efeito da temperatura de armazenamento e da aplicação da cera na conservação pós-colheita de tangerinas 'Fremont' IAC-543. No primeiro experimento, os frutos foram armazenados a 5,10 e $22{ }^{\circ} \mathrm{C}$ por 18 dias, seguido por 18 dias a $22{ }^{\circ} \mathrm{C}$. No segundo experimento, os frutos foram recobertos por cera à base de carnaúba (12\%) e armazenados a $22 \mathrm{e}$ $10^{\circ} \mathrm{C}$ por 18 dias, seguido por 18 dias a $22{ }^{\circ} \mathrm{C}$. A refrigeração reduziu a perda de massa e perda de firmeza e conservou a aparência dos frutos durante o armazenamento. Após a refrigeração, a aparência se manteve boa até o $15^{\circ}$ dia. Tangerinas armazenadas a $22^{\circ} \mathrm{C}$ mantiveram aparência boa até o sexto dia de armazenamento. O recobrimento reduziu a perda de massa e perda de firmeza em frutos armazenados a $22{ }^{\circ} \mathrm{C}$, no entanto prejudicou o sabor dos frutos. $\mathrm{O}$ uso da cera não influenciou a manutenção da firmeza, a aparência ou a perda de massa dos frutos durante a refrigeração, e prejudicou o sabor das tangerinas nesse período. Portanto, tangerinas 'Fremont' IAC-543 podem ser armazenadas a $5{ }^{\circ} \mathrm{C}$ ou a $10{ }^{\circ} \mathrm{C}$ por 18 dias seguido de até 15 dias a $22^{\circ} \mathrm{C}$, sem comprometer a qualidade interna e externa dos frutos. A aplicação de cera à base de carnaúba $(12 \%)$ não tem efeito aditivo à refrigeração de tangerinas 'Fremont' IAC-543.
\end{abstract}

Termos de indexação: recobrimento, conservação, armazenamento refrigerado, citros de mesa.

\section{Cold storage and wax coating on postharvest quality of Fremont tangerine}

\begin{abstract}
Appropriate post-harvest techniques are fundamental for a successful storage and the association of more than one technique can bring up additive effect on maintaining the fruit quality, as for 'Fremont' IAC 543 tangerine, fruit with great potential for commercialization. The objective of this study was to evaluate the effect of storage temperatures and wax application on post-harvest conservation of 'Fremont' IAC 543 tangerines. In the first experiment the fruit were stored at 5,10 and $22{ }^{\circ} \mathrm{C}$ for 18 days, followed by 18 days at $22^{\circ} \mathrm{C}$. In the second one, the fruit were covered with carnauba wax $(12 \%)$ and stored at 10 and $22{ }^{\circ} \mathrm{C}$ for 18 days, followed by 18 days at $22{ }^{\circ} \mathrm{C}$. Refrigeration reduced mass loss and firmness, maintaining fruit appearance appearance during the storage. After refrigeration,

\footnotetext{
${ }^{1}$ Programa de Pós-graduação em Agricultura Tropical e Subtropical, Instituto Agronômico - IAC, Campinas, SP, Brasil

${ }^{2}$ Centro de Ecofisiologia e Biofísica, Instituto Agronômico - IAC, Campinas, SP, Brasil

Autor correspondente: Ilana Urbano Bron, Instituto Agronômico - IAC, Av. Dr. Theodureto de Almeida Camargo, 1500, CEP 13075-630, Campinas, SP, Brasil. E-mail: ilana@iac.sp.gov.br
} 
the appearance remained good until the $15^{\text {th }}$ day. Tangerines stored at $22{ }^{\circ} \mathrm{C}$ maintained good appearance until the $6^{\text {th }}$ day of storage. The application of wax reduced mass loss and firmness in fruit stored at $22{ }^{\circ} \mathrm{C}$, however compromised its flavor. The wax contributed neither for firmness or mass loss nor for the preservation of fruit appearance, and it did depreciate the tangerines flavor in that period. Therefore, 'Fremont' IAC 543 tangerines may be stored at 5 or $10^{\circ} \mathrm{C}$ for 18 days followed by up to 15 days at $22^{\circ} \mathrm{C}$ without compromising the internal and external fruit quality. The application of carnauba wax (12\%) has no addictive effect to cold storage of 'Fremont' IAC 543 tangerines.

Indexing terms: coating, preservation, refrigerated storage, table citrus.

\section{INTRODUÇÃO}

A tangerina 'Fremont' IAC-543 é uma variedade bastante promissora para o mercado de citros de mesa, pois além de apresentar excelente qualidade para consumo in natura, pode ser mantida na planta por um maior período quando comparada à tangerina Ponkan (Citrus reticulata Blanco), possibilitando a ampliação da safra das tangerineiras (Pio et al., 2006). Outra característica importante dessa tangerina é a tolerância à mancha-marrom de Alternaria (Feichetenberger et al., 2005; Pacheco et al., 2012), doença limitante ao cultivo de Ponkan (Citrus reticulata Blanco) principal variedade produzida no país.

A refrigeração é uma das técnicas mais eficientes para a manutenção da qualidade pós-colheita dos frutos. A temperatura recomendada para o armazenamento de tangerinas é de 5 a $8{ }^{\circ} \mathrm{C}$ (Obenland et al., 2013). Para laranjas 'Folha Murcha' o armazenamento a $7^{\circ} \mathrm{C}$ é mais indicado para a conservação dos frutos quando comparado à temperatura de $25^{\circ} \mathrm{C}$ (Todisco et al., 2012) e em tangerinas 'Nadorcotte' a temperatura de $4{ }^{\circ} \mathrm{C}$ mantém a qualidade por 45 dias (Nadello et al., 2017).

A baixa temperatura de armazenamento pode ser ainda mais vantajosa com a associação de recobrimentos. Em citros, o recobrimento com cera é comumente utilizado com a finalidade de disponibilizar ao consumidor frutos com qualidade e boa aparência. A aplicação de cera atua favorecendo o brilho, reduzindo a perda de massa e, portanto, prolongando a vida pós-colheita (Pereira et al., 2014). Porém, o recobrimento pode promover o aparecimento de sabor não característico ou desagradável caso haja fermentação nos frutos (Cohen et al., 1990). Em tangerinas 'Siam Banjar' (Citrus reticulata) a associação entre a aplicação de cera $(12 \%)$ e refrigeração $\left(5^{\circ} \mathrm{C}\right)$ é efetiva em manter a qualidade e estender a vida pós-colheita da fruta (Hassan et al., 2014).

Deste modo, com a projeção de novas variedades para o mercado de citros de mesa, são necessários estudos em pós-colheita visando a aplicação de técnicas eficazes na conservação da qualidade desses frutos. Portanto, o objetivo desse trabalho foi avaliar o efeito da temperatura de armazenamento e da aplicação da cera na conservação pós-colheita de tangerinas 'Fremont' IAC-543.

\section{MATERIAL E MÉTODOS}

\section{Experimento 1: Armazenamento refrigerado}

Frutos de tangerineira 'Fremont' IAC-543 colhidos, com coloração da casca laranja-avermelhado, no mês de maio na safra de 2017 no município de Mogi-Mirim (SP) foram transportados até o laboratório de Pós-Colheita no Centro de Ecofisiologia e Biofísica do Instituto Agronômico (IAC) em Campinas (SP). Os frutos foram armazenados em três temperaturas: $22{ }^{\circ} \mathrm{C} \pm 2{ }^{\circ} \mathrm{C} / 70 \%$ $\pm 2 \%$ UR, $5{ }^{\circ} \mathrm{C} \pm 1{ }^{\circ} \mathrm{C} / 83 \% \pm 3 \%$ UR e $10{ }^{\circ} \mathrm{C} \pm 1{ }^{\circ} \mathrm{C} /$ $86 \% \pm 2 \%$ UR por 18 dias, com análises da qualidade a cada 6 dias, seguidos de transferência para $22^{\circ} \mathrm{C}$ por mais 18 dias com intervalo de três dias entre as análises. $\mathrm{O}$ delineamento estatístico foi inteiramente casualizado em arranjo fatorial (temperaturas $\mathrm{x}$ dias de armazenamento).

\section{Experimento 2: Recobrimento e refrigeração}

Os frutos de tangerineira 'Fremont' IAC-543 foram colhidos em julho na safra de 2017. Depois de lavados em água corrente e secos sob ventilação natural, os frutos receberam aplicação manual de cera à base de carnaúba (12\%) sendo aplicado $0,1 \mathrm{~mL}$ por fruto. Após a aplicação da cera, os frutos foram secos sob ventilação natural. Em seguida, frutos encerados e não encerados foram armazenados a $22{ }^{\circ} \mathrm{C} \pm 1{ }^{\circ} \mathrm{C} / 56 \% \pm 3 \%$ UR e $10{ }^{\circ} \mathrm{C} \pm 1{ }^{\circ} \mathrm{C} / 89 \% \pm 2 \%$ UR durante 18 dias, seguido de transferência (simulação comercial) para $22{ }^{\circ} \mathrm{C} \pm 1 / 61 \% \pm 4,5$ UR por mais 18 dias. Os intervalos entre as análises de qualidade foram os mesmos descritos para o primeiro experimento. O delineamento estatístico foi inteiramente casualizado em esquema fatorial triplo (aplicação de cera $\mathrm{x}$ temperaturas de armazenamento $\mathrm{x}$ dias de armazenamento) para os frutos sob refrigeração e fatorial duplo (aplicação de cera $\mathrm{x}$ dias de armazenamento) após a refrigeração. 


\section{Avaliações}

Firmeza da polpa: avaliada com texturômetro digital (TA-XT2i - Texture Analyser, Stable Micro Systems), com Probe cilíndrico P35, diâmetro de $35 \mathrm{~mm}$, velocidade pré-teste e teste de $2 \mathrm{~mm} \cdot \mathrm{s}^{-1}$ e compressão de $9 \mathrm{~mm}$, tomando-se duas leituras por fruto e os dados expressos em Newtons. Foram usadas dez repetições por tratamento, sendo cada repetição composta por um fruto. Sólidos solúveis: determinado por refratômetro digital (Atago PR-101,) com o suco da polpa e o resultado expresso em ${ }^{\circ}$ Brix. Foram usadas cinco repetições por tratamento, sendo cada repetição composta por dois frutos. Acidez titulável: determinada pela titulação de uma alíquota de $10 \mathrm{~g}$ de suco, o qual foi adicionado $90 \mathrm{ml}$ de água destilada até o ponto de viragem ao $\mathrm{pH} 8,1$ com hidróxido de sódio $(\mathrm{NaOH}) 0,1 \mathrm{~N}$ e o resultado expresso em $\mathrm{g}$ de ácido cítrico $\cdot 100 \mathrm{~mL}$ de suco $^{-1}$. Foram usadas cinco repetições por tratamento, sendo cada repetição composta por dois frutos. Perda de massa determinada por meio da pesagem de um mesmo grupo de frutos em balança semi-analítica, e calculado a partir da diferença entre peso final e inicial, e os resultados expressos em porcentagem. Foram usadas dez repetições por tratamento, sendo cada repetição composta por um fruto. Análise sensorial realizada por teste de aceitabilidade (trinta provadores não treinados) considerando a aparência e sabor do fruto por meio de escala hedônica de 5 pontos - muito bom (nota 5); bom (nota 4); regular (nota 3); ruim (nota 2) e muito ruim (nota 1). Respiração realizada pela leitura da concentração de $\mathrm{CO}_{2}$ com auxílio de analisador de gás DanSensor (modelo CheckMate) de frutos com massa conhecida mantidos em vidros herméticos por 30 minutos e o resultado expresso em $\mathrm{mL}$ de $\mathrm{CO}_{2} \cdot \mathrm{kg}^{-1} \cdot h^{-1}$. Foram usadas sete repetições por tratamento, sendo cada repetição composta por um fruto. Apenas no segundo experimento foi avaliada a luminosidade da casca determinada com colorímetro (Minolta CR-300), tomando-se duas leituras na região equatorial dos frutos. Foram usadas dez repetições por tratamento, sendo cada repetição composta por um fruto.

\section{Análise de dados}

Em ambos os experimentos, os dados obtidos foram submetidos à análise de variância e a comparação de médias foi realizada pelo teste de Tukey à $5 \%$ de probabilidade, utilizando o programa de assistência estatística ASSISTAT (Silva \& Azevedo, 2016).

\section{RESULTADOS}

Experimento 1: Houve diminuição da respiração nos frutos refrigerados a partir do $6^{\circ}$ dia de armazenamento com média de 3,1 e $4,0 \mathrm{~mL}$ de $\mathrm{CO}_{2} \cdot \mathrm{kg}^{-1} \cdot \mathrm{h}^{-1}$ nos frutos mantidos a 5 e $10{ }^{\circ} \mathrm{C}$, respectivamente, permanecendo baixa até o final do período (Figura 1A). Nos frutos armazenados a $22{ }^{\circ} \mathrm{C}$, a respiração que inicialmente era de $24,1 \mathrm{~mL}$ de $\mathrm{CO}_{2} \cdot \mathrm{kg}^{-1} \cdot \mathrm{h}^{-1}$ decresceu gradativamente atingindo valores de $14,3 \mathrm{~mL}$ de $\mathrm{CO}_{2} \cdot \mathrm{kg}^{-1} \cdot \mathrm{h}^{-1}$ aos 6 dias de armazenamento e $6,9 \mathrm{~mL}$ de $\mathrm{CO}_{2} \cdot \mathrm{kg}^{-1} \cdot \mathrm{h}^{-1}$ aos 12 dias (Figura 1A).

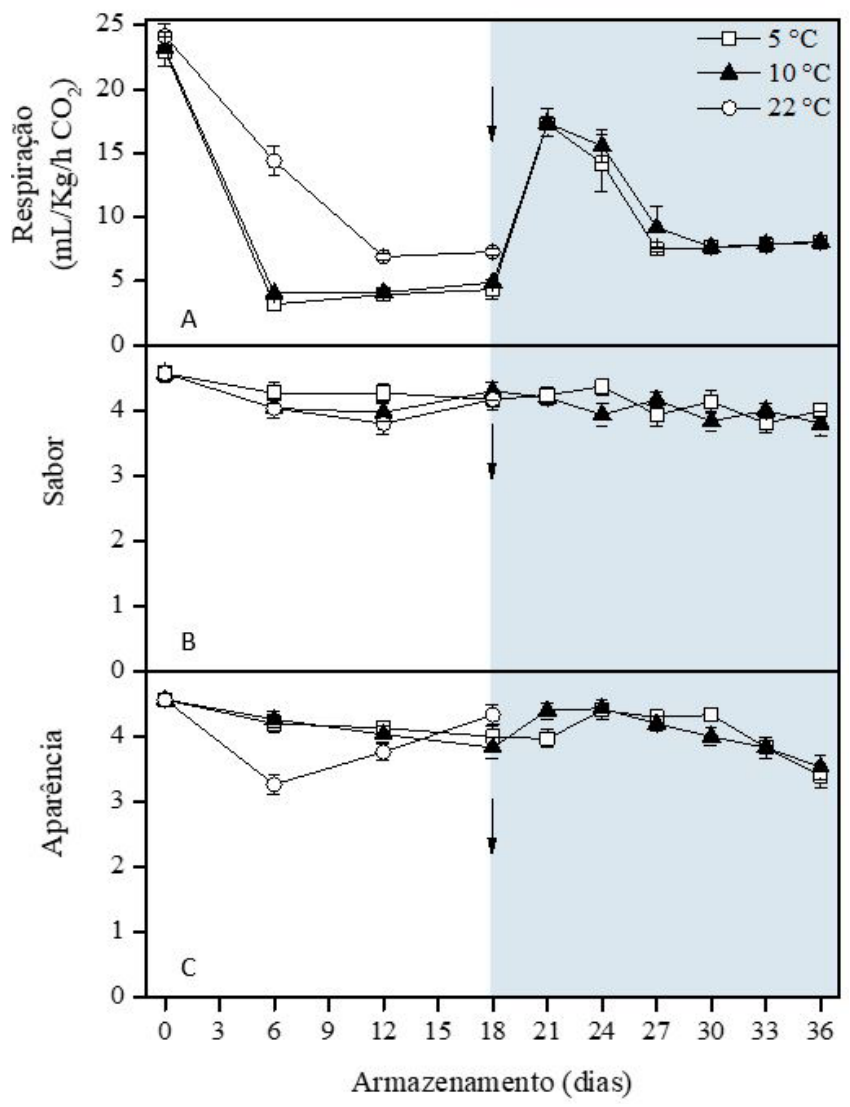

Figura 1. Respiração (A), sabor (B) e aparência (C) de tangerinas 'Fremont' IAC-543 armazenadas a $5{ }^{\circ} \mathrm{C} / 83 \%$ UR, $10{ }^{\circ} \mathrm{C} / 86 \%$ UR e $22^{\circ} \mathrm{C} / 70 \%$ UR, por 18 dias, seguido de transferência para $22^{\circ} \mathrm{C}$ por mais 18 dias. A seta indica o momento exato em que os frutos refrigerados $\left(10\right.$ e $\left.5^{\circ} \mathrm{C}\right)$ foram transferidos para $22^{\circ} \mathrm{C}$. Aárea sombreada representa o período que os frutos, previamente refrigerados, permaneceram a $22^{\circ} \mathrm{C}$. Barras verticais indicam o erro padrão das médias (A - n=7; B, C - n=30). Escala de notas para aparência e sabor: 5 - Muito bom, 4 - Bom, 3 -Regular, 2 - Ruim e 1 - Muito ruim. 
Após o período de armazenamento refrigerado, tanto os frutos armazenados a 5 como a $10{ }^{\circ} \mathrm{C}$ apresentaram aumento na respiração assim que foram retirados da refrigeração. A respiração manteve-se elevada até o sexto dia a $22{ }^{\circ} \mathrm{C}$ quando então decresceu mantendo valores médios de $10,3 \mathrm{~mL}$ de $\mathrm{CO}_{2} \cdot \mathrm{kg}^{-1} \cdot \mathrm{h}^{-1}$ até o final do período estudado (Figura 1A).

Não houve influência da temperatura de armazenamento no sabor das tangerinas, que se manteve com notas próximas de 4 (bom) durante todo período avaliado (Figura 1B) inclusive no período pós-refrigeração, sem diferença significativa entre as temperaturas prévias de refrigeração (Figura 1B).

Arefrigeração manteve as notas de aparência. Aos 18 dias de refrigeração as notas de aparência dos frutos eram de 4 (bom) e 3,8 para os frutos mantidos a 5 e $10{ }^{\circ} \mathrm{C}$, respectivamente. Após o período refrigerado, os frutos mantiveram notas de aparência acima de 4 (bom) até o $15^{\circ}$ dia de armazenamento (Figura $1 \mathrm{C}$ ). Frutos armazenados a $22^{\circ} \mathrm{C}$ tiveram redução na nota de aparência aos 6 dias de armazenamento, essas notas se mantiveram próximas a 3 (regular) até o final do armazenamento (Figura 1C).

Apesar da perda de massa crescente, independentemente da temperatura de armazenamento, frutos refrigerados apresentaram menor perda de massa quando comparados àqueles mantidos a $22{ }^{\circ} \mathrm{C}$. Considerando os 18 dias de armazenamento, frutos refrigerados a 5 e $10{ }^{\circ} \mathrm{C}$ tiveram perda de massa média de $4,2 \%$ e $3,6 \%$, respectivamente, enquanto àqueles mantidos a $22{ }^{\circ} \mathrm{C}$ perderam cerca de $11,1 \%$ de sua massa (Figura 2A). Após o período de refrigeração, a perda de massa também foi crescente e os frutos a $10{ }^{\circ} \mathrm{C}$ apresentaram perda média $(8,5 \%)$ estatisticamente menor em relação aos frutos previamente armazenados a $5{ }^{\circ} \mathrm{C}(9,2 \%)$ (Figura $\left.2 \mathrm{~A}\right)$.

No armazenamento a $22{ }^{\circ} \mathrm{C}$ houve diminuição da firmeza da polpa, principalmente a partir do $12^{\circ}$ dia de armazenamento, aos 18 dias a $22{ }^{\circ} \mathrm{C}$ as tangerinas apresentaram firmeza de 51 N. Durante a refrigeração, os frutos mantiveram a firmeza durante todo o armazenamento com valores de 100,4 e $88,4 \mathrm{~N}$ para aqueles mantidos 5 e $10^{\circ} \mathrm{C}$, respectivamente (Figura $2 \mathrm{~B}$ ). Após a refrigeração, houve uma diminuição significativa na firmeza dos frutos. Frutos anteriormente armazenados a $5{ }^{\circ} \mathrm{C}$ apresentaram média de firmeza significativamente maior $(73,2 \mathrm{~N}) \mathrm{em}$ relação aos frutos a $10{ }^{\circ} \mathrm{C}(64,8 \mathrm{~N})$ (Figura $\left.2 \mathrm{~B}\right)$.

O ratio aumentou ao longo do período de armazenamento, independentemente da temperatura. Tangerinas que no início do armazenamento tinham 12,9 de ratio, apresentaram média de 13,7 aos 18 dias de armazenamento (Figura 2C). No período pós-refrigeração não houve diferença estatística entre os frutos armazenados em ambas temperaturas de refrigeração. A média foi de 13,7 e 13,8 para os frutos a $5^{\circ} \mathrm{C}$ e a $10{ }^{\circ} \mathrm{C}$, respectivamente.

Experimento 2: foi utilizada a temperatura de $10^{\circ} \mathrm{C}$ para o armazenamento refrigerado associado à aplicação de cera, uma vez que, de acordo com o experimento anterior, a perda de massa foi menor após a refrigeração em relação aos frutos previamente armazenados a $5{ }^{\circ} \mathrm{C}$.

A aplicação de cera influenciou a redução da respiração dos frutos armazenados a $22{ }^{\circ} \mathrm{C}$ durante todo o período de armazenamento. Frutos sem a aplicação de cera tiveram respiração média de $16,2 \mathrm{~mL}$ de $\mathrm{CO}_{2} \cdot \mathrm{kg}^{-1} \cdot \mathrm{h}^{-1}$, enquanto que àqueles que receberam o recobrimento apresentaram,

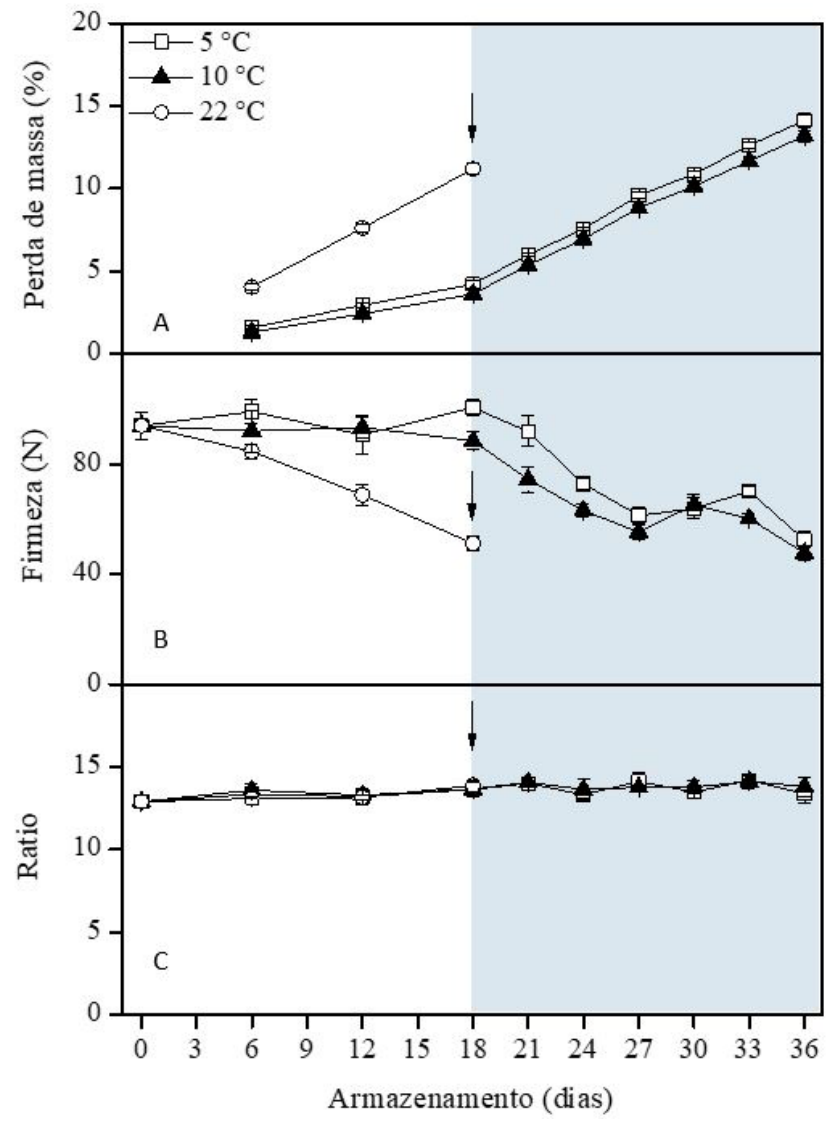

Figura 2. Perda de massa (A), firmeza (B) e ratio (C) de tangerinas 'Fremont' IAC-543 armazenadas a $5{ }^{\circ} \mathrm{C} / 83 \%$ UR, $10{ }^{\circ} \mathrm{C} / 86 \%$ UR e $22{ }^{\circ} \mathrm{C} / 70 \%$ UR, por 18 dias, seguido de transferência para $22{ }^{\circ} \mathrm{C}$ por mais 18 dias. A seta indica o momento exato em que os frutos refrigerados $\left(10\right.$ e $\left.5{ }^{\circ} \mathrm{C}\right)$ foram transferidos para $22^{\circ} \mathrm{C}$. $\mathrm{A}$ área sombreada representa o período que os frutos, previamente refrigerados, permaneceram a $22^{\circ} \mathrm{C}$. Barras verticais indicam o erro padrão das médias $(\mathrm{A}, \mathrm{B}-\mathrm{n}=10$; $\mathrm{C}-\mathrm{n}=5$ ). 
em média, respiração de $5,8 \mathrm{~mL}$ de $\mathrm{CO}_{2} \cdot \mathrm{kg}^{-1} \cdot \mathrm{h}^{-1}$. Durante a refrigeração, o efeito do recobrimento em reduzir a produção de $\mathrm{CO}_{2}$ foi observado no primeiro e no último dia de armazenamento (Figura 3A).

$\mathrm{Na}$ saída da refrigeração, os frutos apresentaram respiração média de $6,2 \mathrm{~mL}$ de $\mathrm{CO}_{2} \cdot \mathrm{kg}^{-1} \cdot \mathrm{h}^{-1}$. Até o $3^{\circ}$ dia de comercialização simulada ocorreu aumento significativo na respiração com média de $13,9 \mathrm{~mL}$ de $\mathrm{CO}_{2} \cdot \mathrm{Kg}^{-1} \cdot \mathrm{h}^{-1}$ seguida por decréscimo até o $15^{\circ}$ dia com média de $9,0 \mathrm{~mL}$ de $\mathrm{CO}_{2} \cdot \mathrm{Kg}^{-1} \cdot \mathrm{h}^{-1}$, independentemente da temperatura de armazenamento prévia. No último dia de armazenamento,

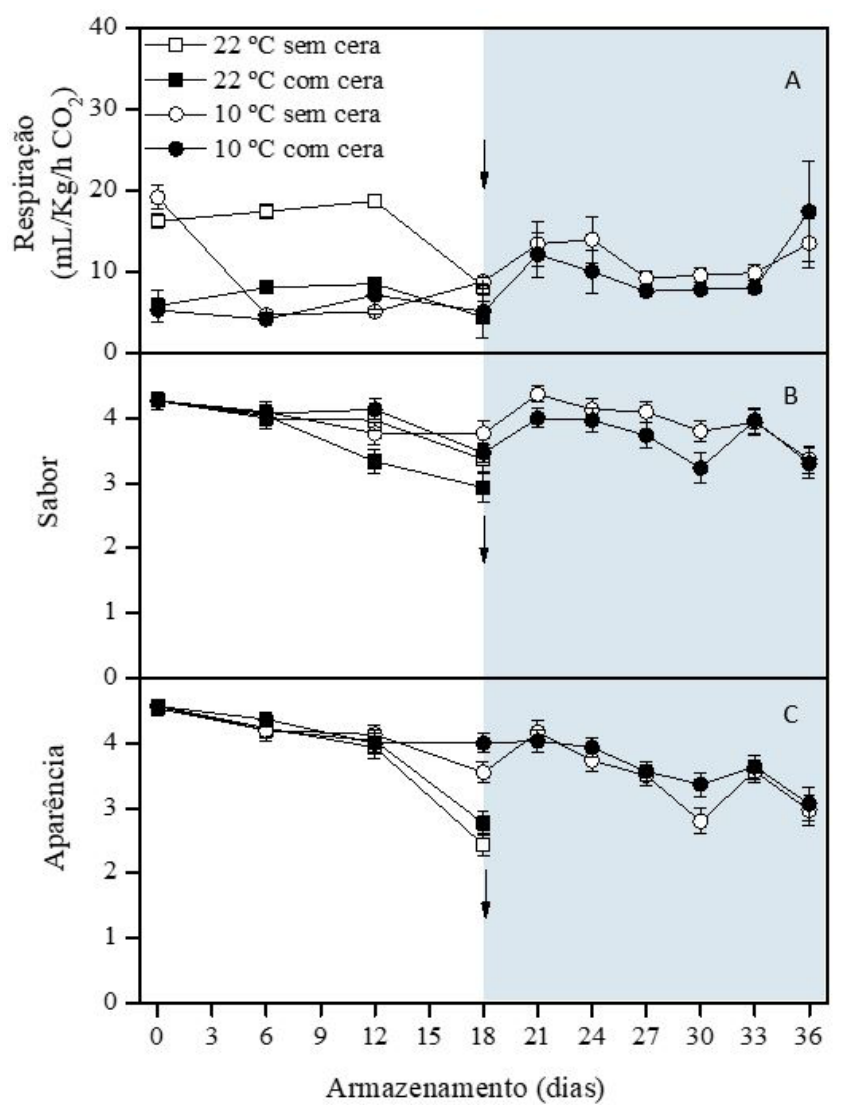

Figura 3. Respiração (A), sabor (B) e aparência (C) de tangerinas 'Fremont' IAC-543 com e sem aplicação de cera à base de carnaúba (12\%) armazenadas a $22^{\circ} \mathrm{C} \pm 1{ }^{\circ} \mathrm{C} / 56 \% \pm 3 \%$ UR e $10{ }^{\circ} \mathrm{C} \pm 1{ }^{\circ} \mathrm{C}$ e $89 \% \pm 2 \%$ UR por 18 dias, seguido de transferência para $22{ }^{\circ} \mathrm{C} \pm 1{ }^{\circ} \mathrm{C} / 61 \% \pm 4,5 \%$ UR por mais 18 dias. A seta indica o momento exato em que os frutos refrigerados $\left(10^{\circ} \mathrm{C}\right)$ foram transferidos para $22{ }^{\circ} \mathrm{C}$. A área sombreada representa o período que os frutos, previamente refrigerados, permaneceram a $22^{\circ} \mathrm{C}$. Barras verticais indicam o erro padrão das médias $(\mathrm{A}-\mathrm{n}=7 ; \mathrm{B}, \mathrm{C}-\mathrm{n}=30)$. Escala de notas para aparência e sabor: 5 - Muito bom, 4 - Bom, 3 - Regular, 2 - Ruim e 1 - Muito ruim. os frutos apresentaram aumento significativo, com média de $15,6 \mathrm{~mL}$ de $\mathrm{CO}_{2} \cdot \mathrm{Kg}^{-1} \cdot \mathrm{h}^{-1}$ (Figura $3 \mathrm{~A}$ ).

Houve influência da aplicação de cera no sabor das tangerinas. A partir do $12^{\circ}$ dia de armazenamento a $22^{\circ} \mathrm{C}$, os frutos foram avaliados com nota média de 3,3 , significativamente inferior aos demais tratamentos, enquanto que os frutos sem aplicação de cera, na mesma temperatura de armazenamento, atingiram a mesma nota somente no final do período avaliado. Os frutos refrigerados e encerados apresentaram alteração no sabor no último dia de refrigeração, com média de 3,4. Os frutos sem o recobrimento mantiveram as notas inalteradas durante todo o período refrigerado sem diferença significativa (Figura 3B). No armazenamento pós-refrigeração, as notas diminuíram no decorrer do período, independentemente da aplicação do recobrimento, porém os frutos que receberam a cera apresentaram média de 3,6, significativamente menor quando comparado aos frutos sem o recobrimento (Figura 3B).

Não foi observado o efeito da aplicação de cera em manter a aparência dos frutos, independentemente da temperatura de armazenamento. A refrigeração a $10{ }^{\circ} \mathrm{C}$ foi eficiente em manter notas próximas a 4 durante todo o período pós-colheita, enquanto que os frutos armazenados a $22^{\circ} \mathrm{C}$, independente da aplicação de cera, conseguiram manter as notas próximas de 4 até o $12^{\circ}$ dia (Figura 3C). Após a refrigeração, a diminuição das notas ocorreu independentemente da aplicação de recobrimento. Até o $3^{\circ}$ dia de armazenamento, as notas foram mantidas próximas a 4, significativamente diferente em relação ao último dia, com média 3 (regular) (Figura 3C).

Houve menor perda de massa nos frutos sob refrigeração a $10{ }^{\circ} \mathrm{C}$, independentemente da aplicação de cera. O recobrimento atuou na redução da perda de massa apenas no armazenamento a $22{ }^{\circ} \mathrm{C}$, quando aos 12 dias de armazenamento, os frutos sem cera apresentaram perda de massa de $10,5 \%$, enquanto que nos frutos com o recobrimento, a perda foi de 6,8\% (Figura 4A). Após a refrigeração, a aplicação de cera também proporcionou menor perda de massa $(10,4 \%)$ em relação aos frutos sem cera $(11,6 \%)$, considerando todo o período de armazenamento (Figura 4A).

A refrigeração manteve a firmeza dos frutos durante todo o armazenamento, independentemente da aplicação do recobrimento. No entanto, a $22^{\circ} \mathrm{C}$ houve menor perda de firmeza nos frutos com aplicação de cera quando comparado com os frutos sem cera a partir do $12^{\circ}$ dia, apresentando firmeza de 57,5 e 39,3 N, respectivamente (Figura 4B). O benefício da cera em diminuir a perda de 


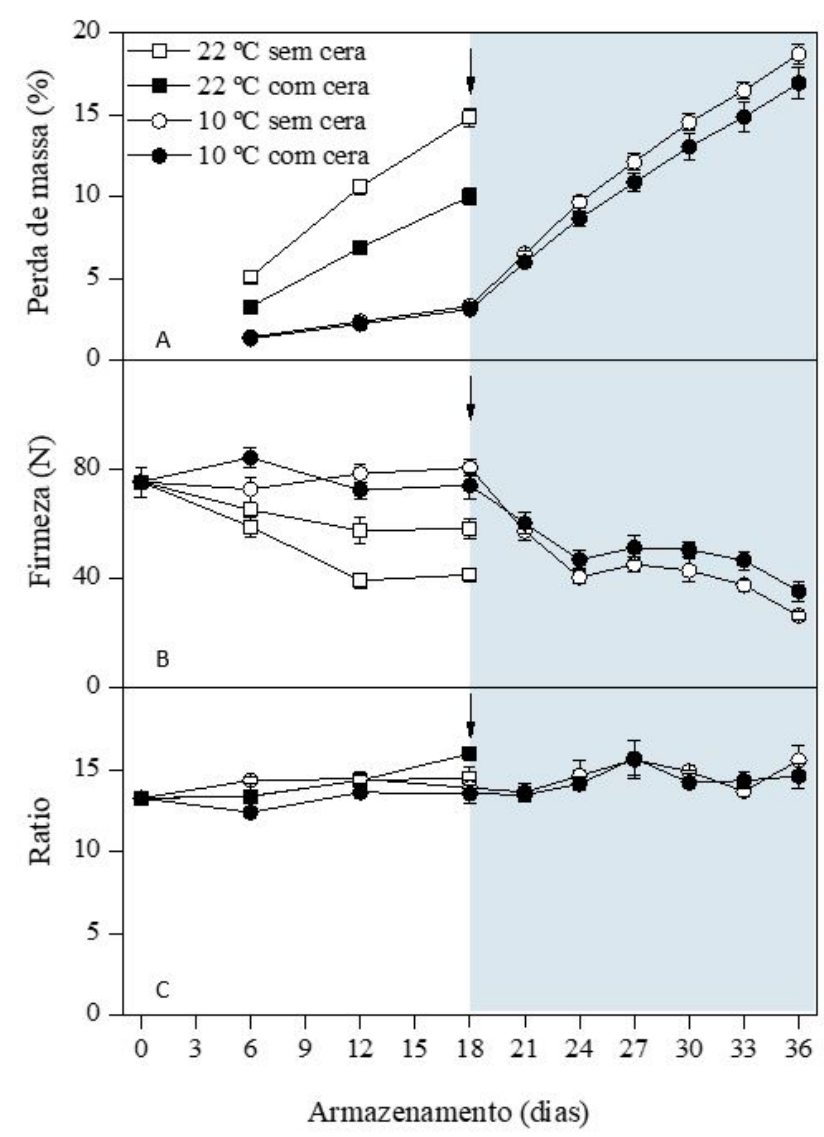

Figura 4. Perda de massa (A), firmeza (B) e ratio (C) de tangerinas 'Fremont' IAC-543 com e sem aplicação de cera à base de carnaúba (12\%) armazenadas a $22{ }^{\circ} \mathrm{C} \pm 1{ }^{\circ} \mathrm{C} / 56 \% \pm 3 \%$ UR e $10{ }^{\circ} \mathrm{C} \pm 1{ }^{\circ} \mathrm{C}$ e $89 \% \pm 2 \%$ UR por 18 dias, seguido de transferência para $22{ }^{\circ} \mathrm{C} \pm 1{ }^{\circ} \mathrm{C} / 61 \% \pm 4,5 \%$ UR por mais 18 dias. A seta indica o momento exato em que os frutos refrigerados $\left(10^{\circ} \mathrm{C}\right.$ ) foram transferidos para $22^{\circ} \mathrm{C}$. A área sombreada representa o período que os frutos, previamente refrigerados, permaneceram a $22{ }^{\circ} \mathrm{C}$. Barras verticais indicam o erro padrão das médias (A, $\mathrm{B}-\mathrm{n}=10 ; \mathrm{C}-\mathrm{n}=5$ ).

firmeza também foi verificado no período pós-refrigeração, com média de 47,2 e 52,2 N nos frutos sem e com aplicação de cera, respectivamente, durante o armazenamento. No entanto, tanto os frutos que receberam a aplicação do recobrimento quanto os que não receberam apresentaram diminuição significativa até o $6^{\circ}$ dia, mantendo-se pelo restante do período sem diferença estatística (Figura 4B).

No decorrer do armazenamento, frutos com aplicação de cera sob refrigeração apresentaram valores de ratio estatisticamente menor (média de 13,2) quando comparados com os frutos sem a aplicação de cera $(13,9)$ e com os frutos encerados a $22{ }^{\circ} \mathrm{C}(14,2)$ (Figura 4C).

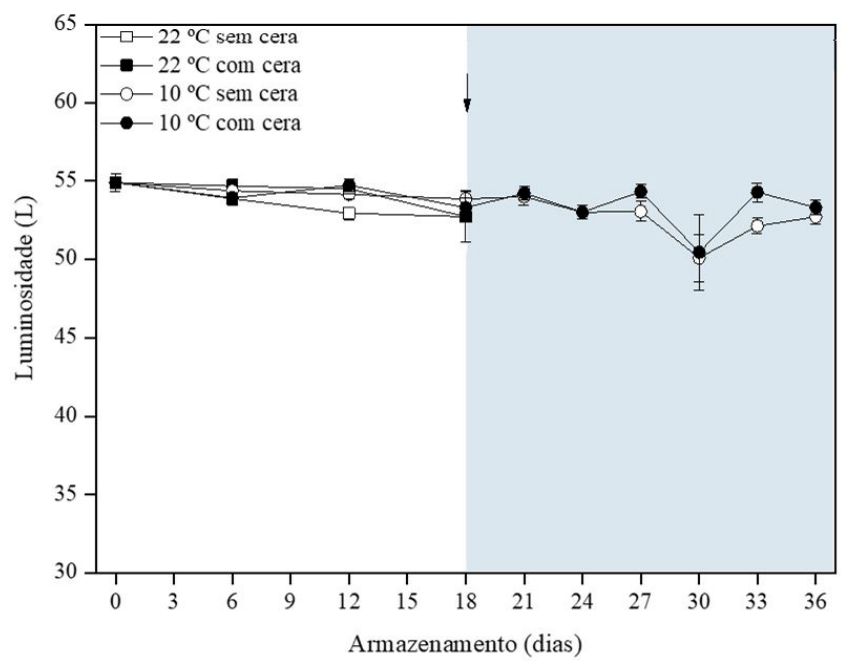

Figura 5. Luminosidade de tangerinas 'Fremont' IAC-543 com e sem aplicação de cera à base de carnaúba $(12 \%)$ armazenadas a $22^{\circ} \mathrm{C} \pm 1{ }^{\circ} \mathrm{C} / 56 \% \pm 3 \%$ UR e $10^{\circ} \mathrm{C} \pm 1{ }^{\circ} \mathrm{C}$ e $89 \% \pm 2 \%$ UR por 18 dias (A) seguidos por mais 18 dias a $22{ }^{\circ} \mathrm{C} \pm 1{ }^{\circ} \mathrm{C} / 61 \% \pm 4,5 \%$ UR (B). Barras verticais representam o erro padrão das médias $(\mathrm{n}=10)$.

Após a refrigeração, a aplicação de cera e o tempo de armazenamento não influenciaram significativamente nos valores de ratio (Figura 4C).

Houve diminuição dos valores de luminosidade da casca no decorrer do armazenamento. Inicialmente os valores de luminosidade eram em média de 54,9 e ao final, a média foi de 53,1 (Figura 5). Após a refrigeração, independentemente da aplicação de cera, os frutos tiveram a luminosidade mantida durante todo o período de armazenamento (Figura 5).

\section{DISCUSSÃO}

O armazenamento refrigerado, independentemente da temperatura utilizada, promoveu redução na respiração (Figura 1A). Também foi observado que a refrigeração retardou a perda de firmeza das tangerinas 'Fremont' IAC-543. As reações enzimáticas ocorrem mais lentamente em temperaturas baixas, reduzindo a respiração e, consequentemente, prolongando a conservação dos frutos em geral. A perda da firmeza está principalmente ligada ao aumento da solubilização das pectinas presentes na parede celular, portanto é esperado que em temperaturas reduzidas, os processos degradativos da parede celular ocorram mais lentamente. A manutenção da integridade da parede celular obtida pela conservação em ambiente 
refrigerado foi evidenciada pela maior firmeza de tangerinas 'Fremont' armazenadas tanto a $5{ }^{\circ} \mathrm{C}$ quanto a $10^{\circ} \mathrm{C}$ (Figura 2B).

$\mathrm{O}$ murchamento também foi reduzido pela menor perda de água em ambiente refrigerado (Figura 2A). Resultado semelhante foi obtido por Todisco et al. (2012) que observaram importante redução da perda de massa em laranjas 'Folha Murcha' refrigeradas a $7^{\circ} \mathrm{C}$ e Pacheco et al. (2016) em tangerinas 'Fremont' armazenadas a $8{ }^{\circ} \mathrm{C}$. A perda de massa está fortemente associada à aparência, que reflete a qualidade externa do fruto. A refrigeração possibilitou a obtenção de notas acima de 4 durante todo o período de armazenamento, com reduzida perda de massa (Figura 1C).

O padrão respiratório de frutos não climatéricos, como os citros, implica em poucas mudanças na maioria dos atributos físico-químicos e sensoriais das frutas, mas a temperatura de armazenamento pode alterar alguns atributos como visto em tangor 'Murcott' por Obenland et al. (2011) quando o armazenamento a $8{ }^{\circ} \mathrm{C}$ resultou em melhor sabor, quando comparado ao armazenamento a $0{ }^{\circ} \mathrm{C}$ ou a $4{ }^{\circ} \mathrm{C}$. Neste estudo, foi visto que o sabor de tangerinas 'Fremont'IAC-543 se manteve bom, com notas acima de 4 em praticamente todo o período avaliado, independentemente da temperatura de armazenamento (Figura 1B) assim como os valores obtidos para o ratio (Figura 2C). A manutenção do sabor também foi relatada por Tietel et al. (2012) em tangerinas 'Or' ' 'Odem' (Citrus reticulata Blanco) armazenadas em temperaturas de $2^{\circ} \mathrm{C} \mathrm{a}$ $8^{\circ} \mathrm{C}$. Após a refrigeração, as notas do sabor em tangerinas 'Fremont' se mantiveram acima de 4, durante praticamente todo o armazenamento (Figura 1B), demonstrando que esse atributo não foi influenciado também no período de comercialização simulada.

A aparência dos frutos, após o período de refrigeração, foi mantida com notas próximas de 4 até o $15^{\circ}$ dia (Figura 1C). Nesse dia, a perda de massa era de 12,6 e $11,6 \%$ (Figura 2A) e a firmeza de 70,1 e $60,1 \mathrm{~N}$ (Figura 2B) nos frutos previamente armazenados a $5^{\circ} \mathrm{C}$ e $10{ }^{\circ} \mathrm{C}$, respectivamente. Mesmo com o aumento da perda de massa e diminuição da firmeza, a aparência das tangerinas não foi afetada, ou seja, após a refrigeração, somente perda de massa superior a $12 \%$ é prejudicial à aparência de tangerinas 'Fremont' (Figura 1C).

Quando avaliada a associação de cera com refrigeração verificou-se menores valores de ratio, sem alterações significativas na luminosidade, perda de massa e firmeza dos frutos (Figura 4C, 4A, 4B, 5). A contribuição da aplicação de cera foi mais evidente naqueles frutos que permaneceram 18 dias a $22{ }^{\circ} \mathrm{C}$, nessas condições os frutos com aplicação de cera apresentaram maior firmeza, principalmente a partir do $12^{\circ}$ dia.

Para Pereira et al. (2014), a vida pós-colheita de laranjas 'Valência Delta' recobertas com cera a base de carnaúba pode ser prolongada por até 12 dias, com perda de massa de no máximo 6\%. Quando consideramos este valor como aceitável, a cera influenciou positivamente nos resultados para as tangerinas 'Fremont' armazenadas a $22^{\circ} \mathrm{C}$, estendendo em até seis dias sua vida útil, quando considerada somente a perda de massa (Figura 4A). A perda de massa dos frutos ocorre pelo processo de transpiração como consequência do gradiente de pressão de vapor entre o fruto e o ambiente. O recobrimento proporciona a modificação da atmosfera evitando a perda de umidade (Pereira et al., 2014). Cantillano et al., (2011) avaliaram a aplicação de cera à base de carnaúba em tangerinas 'Ponkan' e identificaram, por microscopia eletrônica, homogeneidade na cobertura do flavedo do fruto, proporcionando uma barreira física contra a perda de água e evitando a penetração de patógenos pelas aberturas naturais, devido ao selamento da casca promovido pela cera.

Após o período de refrigeração poucas alterações foram verificadas na qualidade dos frutos. Em relação à perda de massa, a aplicação de cera possibilitou menor perda em relação aos frutos sem recobrimento, no entanto, a partir do $9^{\circ}$ dia, independentemente da aplicação de cera, os frutos apresentaram perda de massa maior que $10 \%$ (Figura 4A).

Apesar do benefício da cera na redução da perda de massa e firmeza a $22{ }^{\circ} \mathrm{C}$, houve prejuízo no sabor dos frutos a partir do $6^{\circ}$ dia de armazenamento com notas inferiores a 4 (bom). Já nos frutos sem o recobrimento, as notas 4 foram mantidas até o $12^{\circ}$ dia, nessa mesma condição de armazenamento (Figura 3B). Jomori et al. (2003) verificaram a presença de sabor e odor estanhos em lima ácida 'Tahiti' com aplicação de cera após 30 dias de armazenamento a $10{ }^{\circ} \mathrm{C}$. Pacheco et al. (2016) observaram notas inferiores para o sabor em tangerinas 'Fremont' com aplicação de cera na primeira semana de armazenamento a $21{ }^{\circ} \mathrm{C}$. Mesmo após a refrigeração, a aplicação de cera prejudicou o sabor dos frutos previamente refrigerados, sendo que, no $9^{\circ}$ dia de armazenamento pósrefrigeração, as notas já estavam abaixo de 4 , enquanto que nos frutos sem recobrimento a nota 4 foi verificada até o $15^{\circ}$ de armazenamento (Figura 3B).

A respiração de tangerinas 'Fremont' IAC-543 foi menor nos frutos com a aplicação de cera a $22{ }^{\circ} \mathrm{C}$ e nos frutos armazenados sob refrigeração (Figura 3A). 
As alterações no sabor evidenciadas nos frutos que receberam a aplicação do recobrimento a $22{ }^{\circ} \mathrm{C}$ pode ter sido ocasionada pela redução da permeabilidade aos gases proporcionada pela aplicação de cera (Hagenmaier, 2002). O sabor desagradável pode estar relacionado à respiração anaeróbica, devido à fermentação promovida em condições de alta concentração de $\mathrm{CO}_{2}$ e baixa de $\mathrm{O}_{2}$.

A luminosidade da casca não foi influenciada pela aplicação de cera independentemente da temperatura de armazenamento (Figura 5). Em laranjas 'Valência Delta', a luminosidade da casca aumentou com aplicação de recobrimentos durante o armazenamento a $24^{\circ} \mathrm{C}$, como observado por Pereira et al. (2014).

Não houve diferenças significativas entre as temperaturas de armazenamento, sendo assim, o uso de temperatura de $10^{\circ} \mathrm{C}$ é o mais racional quando se avalia o custo/benefício para a conservação dessa variedade. Nessas condições, a 'Fremont' foi conservada por 18 dias, e ainda permaneceu por mais 15 dias a $22{ }^{\circ} \mathrm{C} \mathrm{com}$ boa qualidade, o que evidencia o bom potencial de conservação dessa variedade. No que diz respeito ao uso de cera, mais estudos devem ser conduzidos para essa variedade, uma vez que a cera à base de carnaúba (12\%) causou fermentação nos frutos.

\section{CONCLUSÃO}

A tangerina 'Fremont' pôde ser armazenada a $5{ }^{\circ} \mathrm{C}$ ou $10^{\circ} \mathrm{C}$ por 18 dias, seguido de transferência para temperatura de $22{ }^{\circ} \mathrm{C}$ por 15 dias, sem comprometer a qualidade interna e externa dos frutos.

A aplicação de cera à base de carnaúba (12\%) não teve efeito aditivo à refrigeração de tangerinas 'Fremont' mantidas a $10{ }^{\circ} \mathrm{C}$ por 18 dias e, apesar de reduzir a perda de massa e firmeza, prejudicou o sabor dos frutos durante a comercialização simulada a $22{ }^{\circ} \mathrm{C}$.

\section{AGRADECIMENTOS}

À CAPES pela concessão da bolsa de estudo. À Yolanda Eugênia Álamo Gabrine Boza pela contribuição na realização do experimento.

\section{REFERÊNCIAS}

Cantillano, R. F. F., Galarça, S. P., Treptow, R. O., \& Castro, L. A. S. (2011) Efeito da atmosfera modificada na qualidade pós-colheita tangerinas 'PONKAN'durante o armazenamento refrigerado (36 pp.). Pelotas: Embrapa Clima Temperado.

Cohen, E., Shalom, Y., \& Rosenberger, I. (1990). Postharvest ethanol buildup and off-flavor in 'Murcott' tangerine fruits. Journal of the American Society for Horticultural Science, 115(5), 775-778.

Feichetenberger, E., Spósito, M. B., Pio, R. M., \& Castro, J. L. (2005). Seleção de híbridos de tangerinas e híbridos de citros para tolerância à mancha-marrom de alternaria (Alternaria alternata Keissler). Citricultura Atual, 8, 8-10.

Hagenmaier, R. D. (2002). The flavor of mandarin hybrids with different coatings. Postharvest Biology and Technology, 24, 79-87.

Hassan, Z. H., Lesmayati, S., Qomariah, R., \& Hasbianto, A. (2014). Effects of wax coating applications and storage temperatures on the quality of tangerine citrus (Citrus reticulata) var. Siam Banjar. International Food Research Journal, 21(2), 641-648.

Jomori, M. L. L., Kluge, R. A., Jacomino, A. P., \& Tavares, S. (2003). Conservação refrigerada de lima ácida 'Tahiti': Uso de 1-Metilciclopropeno, ácido giberélico e cera. Revista Brasileira de Fruticultura, 25, 406-409.

Nadello, I. C., Cantillano, R. F. F., Seifert, M., MelloFarias, P. C., Malgarim, M. B., \& Oliveira, R. P. (2017). Postharvest quality during refrigerated storage of 'Nadorcott' mandarin. J19, (5), 1-10.

Obenland, D., Collin, S., Mackey, B., Sievert, J., \& Arpaia, M. L. (2011). Storage temperature and time influences sensory quality of mandarins by altering soluble solids, acidity and aroma volatile composition. Postharvest Biology and Technology, 59, 187-193.

Obenland, D., Collin, S., Sievert, J., \& Arpaia, M. L. (2013). Mandarin flavor and aroma volatile composition are strongly influenced by holding temperature. Postharvest Biology and Technology, 82, 6-14.

Pacheco, C. A., Martelli, I. B., Polydoro, D. A., Schinor, E. H., Pio, R. M., Jupper, K. C., \& Azevedo, F. A. (2012). Resistance and susceptibility of mandarins and their hybrids to Alternaria alternata. Scientia Agrícola, 69, 386-392. 
Pacheco, C. A., Schinor, E. H., Cristofani-ali, M., Machado, M. A., \& Azevedo, F. A. (2016). Fremont mandarin: Fruit with a long shelf life for the fresh fruit market. African Journal of Agricultural Research, 11, 4600-4609.

Pereira, G. S., Machado, F. L. C., \& Costa, J. M. C. (2014). Aplicação de recobrimento prolonga a qualidade póscolheita de laranja 'Valência Delta' durante armazenamento ambiente. Ciência Agronômica, 45, 520-527.

Pio, R. M., Azevedo, F. A., Negri, J. D., Figueiredo, J. O., \& Castro, J. P. (2006). Características da variedade Fremont quando comparas com as tangerinas 'Ponkan' e 'Clementina Nules'. Revista Brasileira de Fruticultura, 28, 222-226.

Silva, F. A. S., \& Azevedo, C. A. V. (2016). The assistat software version 7.7 and its use in the analysis experimental data. African Journal of Agricultural Research, 11, 3733-3740.
Tietel, Z., Lewinsohn, E., Fallik, E., \& Porat, R. (2012). Importance of storage temperatures in maintaining flavor and quality of mandarins. Postharvest Biology and Technology, 64, 175-182.

Todisco, K. M., Clemente, E., \& Rosa, C. I. L. F. (2012). Conservação e qualidade pós-colheita de laranjas 'Folha Murcha' armazenadas em duas temperaturas. Revista em Agronegócios e Meio Ambiente, 5(3), 579-591.

Recebido: Fevereiro 05, 2019 Aceito: Março 10, 2020

Como citar: Barbará, M. A., Veiga, J. C., Silva, B. M. P., Valentini, S. R. T., Cia, P., \& Bron, I. U. (2020). Refrigeração e cera na qualidade pós-colheita da tangerina 'Fremont' IAC-543. Citrus Research \& Technology, 41, e1055. https://doi.org/10.4322/crt.18019 
